\title{
Características de pacientes con alta clínica post SARS-Cov-2 hospitalizados en EsSalud. Región Lambayeque
}

\section{Characteristics of clinic discharge patients with SARS-Cov-2 hospitalized at EsSalud in the Lambayeque Region}

Rosario Alexandra Soto- Cáceres Cabanillas ${ }^{1,2, a}$, Cynthia Ivonne TerronesHuamán $^{1,2, a}$, Víctor Alberto Soto-Cáceres ${ }^{2, \mathrm{~b}}$, Cristian Díaz-Vélez ${ }^{1,3, \mathrm{c}}$

\begin{abstract}
RESUMEN
Introducción. La pandemia por Covid 19 ha ocasionado alta letalidad en la región Lambayeque colapsando los hospitales. Objetivos: Describir según genero las características de pacientes con alta clínica post Sars. Cov2 hospitalizados en Essalud de la Región Lambayeque. Material y Métodos: Estudio observacional, retrospectivo, descriptivo, seleccionando una muestra representativa de los casos confirmados COVID 19 entre el 01 de abril al 30 de junio del 2020 en el Hospital Nacional Almanzor Aguinaga Asenjo (HNAAA) y Hospital Luis Heysen Inchaustegui (HLHI); como criterios de exclusión: Aquellos con menos de 48 horas de estancia, los fallecidos y los referidos a otros establecimientos. Se recolectaron los datos de historia clínica en el sistema SGSS, se utilizó el software Excel para el procesamiento de datos. Resultados: se registraron 329 pacientes de los cuales $70 \%$ fueron varones, promedio de edad 55,9 + / - 11 años; el diagnóstico fue mediante prueba serológica $80 \%$ y Tomografía de tórax $17 \%$. La disnea fue el síntoma predominante $85 \%$, linfopenia $51 \%$ y proteína C reactiva alta $39 \%$ y $\mathrm{LDH}$ alta $35 \%$. El tratamiento fue principalmente oxígeno $89 \%$ y enoxaparina $87 \%$, azitromicina $86 \%$ y ceftriaxona $70 \%$, las complicaciones hospitalarias fueron el SDRA 88, $1 \%$ y la neumonía atípica 86,6\%, la estancia en hospitalización fue de 7,85 +/- 5,42 días. Conclusiones: La mayoría de hospitalizados en Essalud fueron varones, promedio edad 55, 9 años; síntoma principal disnea; predominó la linfopenia con proteína C reactiva y LDH altas; el tratamiento fue en base a oxígeno, y enoxaparina, promedio de estancia en hospitalización de 7,85 días.
\end{abstract}

Palabras Clave: Infecciones por Coronavirus; factores epidemiológicos; manifestaciones clínicas; terapéutica; tiempo de permanencia Fuente: DeCS-BIREME).

\begin{abstract}
Introduction. The Covid 19 pandemic has caused high lethality in the Lambayeque region, collapsing hospitals. Objectives: describe, by gender, the characteristics of clinic discharge patients with sarscov2 hospitalized at EsSalud in the Lambayeque region. Material and Methods: Observational, retrospective, descriptive study, selecting a representative sample of confirmed COVID 19 cases between April 1 to June 30, 2020 at the Almanzor Aguinaga Asenjo National Hospital (HNAAA) and the Luis Heysen Inchaustegui Hospital $(\mathrm{HLHI})$; as exclusion criteria: those with less than 48

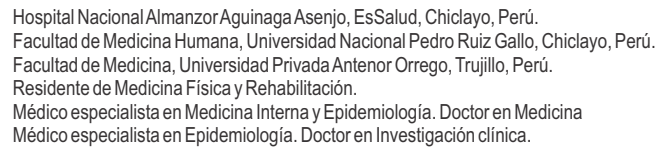

hours of stay, the deceased and those referred to other establishments. The clinical history data were collected in the SGSS system, Excel software was used for data processing. Results: 329 patients were registered, of which $70 \%$ were male, mean age $55.9+/$ 11 years; Diagnosis was by serological test $80 \%$ and chest tomography $17 \%$. Dyspnea was the predominant symptom $85 \%$, lymphopenia $51 \%$ and high C-reactive protein $39 \%$ and high LDH 35\%. Treatment was mainly oxygen $89 \%$ and enoxaparin $87 \%$, azithromycin $86 \%$ and ceftriaxone $70 \%$, hospital complications were ARDS $88.1 \%$ and atypical pneumonia $86.6 \%$, hospital stay was $7.85+/$ - 5.42 days. Conclusions: The majority of hospitalized in Essalud were men, average age 55.9 years; main symptom dyspnea; lymphopenia with high C-reactive protein and LDH predominated; the treatment was based on oxygen and enoxaparin, mean hospital stay of 7.85 days. 
Keywords: Coronavirus infection; signs and synptoms; epidemiologic factors; therapeutics; residence time (Source: DeCS-BIREME).

\section{INTRODUCCIÓN}

En diciembre del 2019, un total de 41 casos de neumonía de etiología desconocida habían sido confirmados en la ciudad de Wuhan, provincia de Hubei, China. La ciudad de Wuhan es un importante centro de transporte con una población de más de 11 millones de personas. En una conferencia de prensa nacional celebrada en enero del 2020, el Dr. Jianguo Xu, académico de Ingeniería de China, anunció que un nuevo coronavirus, nombrado provisionalmente por la Organización Mundial de la Salud como el nuevo coronavirus 2019 (2019-nCoV), había causado este brote $^{(1)}$.

Este nuevo coronavirus tiene un ácido nucleico específico de coronavirus diferente a la secuencia de especies conocidas de coronavirus humanos, que son similares a algunos de los beta coronavirus identificados en los murciélagos; las secuencias de ácido nucleico específicas del virus se detectaron en muestras de líquido pulmonar, muestras de sangre y de garganta en 15 pacientes y el virus que se aisló mostró una apariencia típica de coronavirus bajo microscopía electrónica. En los humanos, los coronavirus se encuentran entre el espectro de virus que causan el resfriado común y la enfermedad respiratoria más grave, específicamente el SARS y el MERS, que son ambas zoonosis ${ }^{(2)}$.

El número de casos aumentó rápidamente en el resto de Hubei y se propagó a otros territorios. La rápida expansión de la enfermedad hizo que la Organización Mundial de la Salud, el 30 de enero de 2020, la declarara una emergencia sanitaria de preocupación internacional, basándose en el impacto que el virus podría tener en países subdesarrollados con menos infraestructuras sanitarias y la reconociera como una pandemia el 11 de marzo ${ }^{(2)}$.

Esta enfermedad se caracteriza por producir neumonía severa progresiva y una tormenta de citoquinas, se extendió del Asia a Italia, España, Francia y Reino Unido. El primer caso reportado en América fue en Estados Unidos de Norteamérica (EEUU) el 23 de enero de 2020, pero recién a fines de marzo el número de casos se incrementa abruptamente y en América del Sur se presentó primero en Argentina en febrero de 2020, siendo actualmente los países más afectados de esta parte del continente: Brasil, Chile, Ecuador y Perú(2).

En nuestro país, se confirmó la primera persona infectada el 6 de marzo de 2020, incrementándose rápidamente el número de casos, se presentan las primeras víctimas mortales el 19 de marzo; un estudio revisa 14 casos confirmados, diagnosticados por RTPCR, fallecidos hasta el 04 de abril en el Hospital Edgardo Rebagliati precisando 78,6\% varones, edad promedio 73,4 años con factores de riesgos, síntomas de disnea, fiebre y tos, proteína $C$ reactiva elevada $\mathrm{e}$ infiltrado pulmonar bilateral en vidrio esmerilado, en ventilación mecánica $78,6 \%$, estancia hospitalaria promedio de 4,7 días ${ }^{(3)}$.

Wang D, y colaboradores (Wuhan, China) realizó serie de casos retrospectivos con una población de 138 pacientes en el Hospital Zhongnan de la Universidad de Wuhan, donde se describe que la edad media fue de 56 años, 54,3\% fueron hombres. Los síntomas comunes incluyeron fiebre $98,6 \%$, fatiga $69,6 \%$ y tos seca $59,4 \%$. Linfopenia $70,3 \%$, tiempo prolongado de protrombina $58 \%$ y lactato deshidrogenasa elevada $39,9 \%$. Las tomografías computarizadas de tórax mostraron sombras irregulares bilaterales $u$ opacidad de vidrio esmerilado en los pulmones de todos los pacientes. La mayoría de los pacientes recibieron terapia antiviral oseltamivir $89,9 \%$ moxifloxacina $64,4 \%$ corticoides $44,9 \%$. El tiempo medio desde el primer síntoma hasta la disnea fue de 5 días, el ingreso hospitalario fue de 7 días y el SDRA fue de 8 días. Los pacientes tratados en la $\mathrm{UCI}(\mathrm{n}=36)$, en comparación con los pacientes no tratados en la $\mathrm{UCI}(\mathrm{n}=102)$, eran mayores (edad media, 66 años frente a 51 años), comorbilidades $72,2 \%$ vs $37,3 \%, 34,1 \%$ fueron dados de alta y 6 fallecieron (mortalidad general, $4,3 \%)^{(4)}$.

En Perú hasta el 30 de junio del 2020 se han procesado muestras para 1'679,386 personas por Covid-19, obteniéndose, 285,213 resultados positivos para SARSCoV-2, 11049 pacientes están hospitalizados, 1185 en cuidados intensivos con ventilación mecánica y se habían reportado 9677 fallecimientos ${ }^{(5)}$.

En la región Lambayeque el reporte COVID 19 de la Gerencia Regional de Salud al 21 de junio indicaba 12,315 casos positivos: 10,470 por prueba serológica y 1 845 prueba molecular y 901 fallecidos con una tasa de letalidad de 7,31\% casi el doble del promedio nacional de ese momento ${ }^{(6)}$.

No existen estudios referentes a las características clínicas y epidemiológicas de los pacientes posterior a la hospitalización en la Región Lambayeque se planteó el objetivo de describir las características de pacientes con síndrome post SARS-CoV-2 hospitalizados en región Lambayeque en el periodo abril-junio 2020.

\section{MATERIAL Y METODOS}

Se realizó un estudio de tipo cuantitativo, observacional, retrospectivo y descriptivo en dos hospitales de la región Lambayeque, Red Asistencial 
Lambayeque de Essalud en el período del 01 de abril al 30 de junio del 2020 en el Hospital Nacional Almanzor Aguinaga Asenjo (HNAAA) y Hospital Luis Heysen Inchaustegui (HLHI) según reportes de la Oficina de Inteligencia Sanitaria (OIS) de la Red Asistencial Lambayeque (RAL).

La población total de pacientes confirmados con COVID 19 que fueron dados de alta en ese período y que fueron registrados por la OIS fue de 837 casos de los cuales fueron descartados 201 por presentar registros de hospitalización por motivos administrativos de medicación o de pruebas diagnósticas y otras que se encontraron repetidas en la base datos (transferencias entre servicios o referencias entre hospitales); del total de 636 casos, se estimó el tamaño de la muestra mediante uso del epidat v.3.1 con $95 \%$ de confianza, precisión de $4 \%$, proporción esperada de $50 \%$ para la selección de los casos se utilizó un muestreo probabilístico tipo sistemático basado en la relación existente según fecha de ingreso a hospitalización hasta completar el tamaño de muestra.

\section{Criterios de inclusión}

Pacientes confirmados SARS-Cov-2 con estancia hospitalaria el periodo del 01 de abril al 30 de junio en los dos hospitales Essalud de la Región Lambayeque.

\section{Criterios de exclusión}

- Pacientes con registros de hospitalización por motivos administrativos de medicación o de pruebas diagnósticas y otras que se encontraron repetidas en la base datos (transferencias entre servicios 0 referencias entre hospitales diferentes a essalud).

- Pacientes fallecidos durante su estancia hospitalaria.

- Pacientes que cuenten con un registro ilegible o datos incompletos en el sistema SGSS.

Al ser una muestra representativa los resultados son extrapolables a poblaciones de hospitales de seguridad salud con pacientes diagnosticados de Covid 19.

\section{Definiciones operacionales \\ Paciente covid19:}

Paciente sospechoso diagnosticado mediante prueba molecular PCR o prueba serológica IgM o tomografía de tórax compatible con complicación Covid-19.

\section{Hospitalización:}

Paciente ingresado a cualquier piso de hospitalización por el diagnóstico de COVID19 y que se queda internado más de dos días (48 horas).

\section{Observación de emergencia:}

Paciente ingresado a las áreas de observación de emergencia de donde, en su momento, será ingresado a hospitalización o dado de alta para su domicilio o referido a otro establecimiento.

\section{Alta:}

Procedimiento por medio del cual se indica que el paciente sale de la hospitalización cualquiera sea el servicio donde figure, y regresa a su domicilio.

\section{Instrumentos y procedimientos de recolección de} datos:

Los datos fueron obtenidos del registro de historia clínica en el sistema SGSS, consultando la historia clínica en físico de ser necesario, a través de una ficha de recolección de datos.

El instrumento usado fue una ficha de recolección de datos principales y base de datos sistema COVID red asistencial Lambayeque de abril a junio, el mismo que por su naturaleza de obtención de datos secundarios no requirió validación.

Para el procesamiento y análisis de la información se utilizó la estadística descriptiva, se elaboraron tablas simples y de doble entrada. Se utilizó el software EXCEL para el registro y análisis de datos.

\section{Aspectos éticos}

El estudio siguió las pautas éticas internacionales de investigación haciendo prevalecer los principios bioéticos de autonomía, beneficencia y no maleficencia.

Dado que se trata de un estudio observacional con información secundaria fundamentalmente de archivos, historia clínica se guardará confidencialidad en los datos utilizando códigos para su registro en la ficha correspondiente. Fue revisado por el comité de ética e investigación y aprobado por la Oficina de inteligencia Sanitaria para el acceso a la información.

\section{RESULTADOS}

Los pacientes hospitalizados con diagnóstico COVID 19 en dos hospitales de EsSalud en el período abril-junio y salieron de alta fueron 329 de los cuales el $70 \%$ fueron varones, mayores de 60 años, el promedio de edad fue $55,9+/$ - 10,99 años; el 41\%, provenientes en su mayoría de Chiclayo metropolitano $66 \%$, la confirmación de la 
Tabla 1. Características de los pacientes diagnosticados con Sars-Cov2 hospitalizados en EsSalud en región Lambayeque. abril-junio 2020.

\begin{tabular}{|c|c|c|}
\hline Caracteristicas & $N=329$ & $\%$ \\
\hline \multicolumn{3}{|l|}{ Sexo } \\
\hline Masculino & 230 & $70 \%$ \\
\hline Femenino & 99 & $30 \%$ \\
\hline \multicolumn{3}{|l|}{ Grupo de Edad } \\
\hline 20-29años & 6 & $2 \%$ \\
\hline 30-39 años & 34 & $10 \%$ \\
\hline 40-49 años & 76 & $23 \%$ \\
\hline 50-59 años & 78 & $24 \%$ \\
\hline 60-69 años & 85 & $26 \%$ \\
\hline 70-79 años & 34 & $10 \%$ \\
\hline 80- màs años & 16 & $5 \%$ \\
\hline \multicolumn{3}{|l|}{ Examen de Confirmación } \\
\hline PCR - Hisopado & 10 & $3 \%$ \\
\hline Prueba Serológica & 263 & $80 \%$ \\
\hline TEM Tórax & 56 & $17 \%$ \\
\hline Comorbilidad & $\mathrm{N}$ & \\
\hline HTA & 85 & $25,84 \%$ \\
\hline Diabetes & 61 & $18,54 \%$ \\
\hline Obesidad & 45 & $13,68 \%$ \\
\hline Asma & 13 & $3,95 \%$ \\
\hline Otras enfermedades Cardiovasculares & 13 & $3,95 \%$ \\
\hline Cáncer & 8 & $2,43 \%$ \\
\hline EPOC & 3 & $0,91 \%$ \\
\hline Embarazo & 2 & $0,61 \%$ \\
\hline Enfermedad Renal Crónica & 1 & $0,30 \%$ \\
\hline VIH & 1 & $0,30 \%$ \\
\hline Ninguno & 97 & $29,48 \%$ \\
\hline \multicolumn{3}{|l|}{ DISTRITO } \\
\hline Chiclayo & 150 & $46 \%$ \\
\hline José Leonardo Ortiz & 38 & $12 \%$ \\
\hline La Victoria & 27 & $8 \%$ \\
\hline Ferreñafe & 24 & $7 \%$ \\
\hline Lambayeque & 19 & $6 \%$ \\
\hline Motupe & 10 & $3 \%$ \\
\hline Patapo & 7 & $2 \%$ \\
\hline Otros region Lamb. & 30 & $9 \%$ \\
\hline Otras regiones & 24 & $7 \%$ \\
\hline
\end{tabular}

La disnea fue el síntoma predominante tanto al ingreso como durante la hospitalización $85-86 \%$, la tos, malestar general y fiebre se presentaron antes del ingreso y taquipnea $72 \%$, linfopenia $51 \%$ y proteína C reactiva alta $39 \%$ predominaron durante la hospitalización (Tabla 2).
Tabla 2. Signos, síntomas y analítica de laboratorio según genero de pacientes diagnosticados con sarscov 2 hospitalizados en EsSalud en región Lambayeque. abril-junio 2020.

\begin{tabular}{lcc}
\hline \multicolumn{1}{c}{ Signos y Síntomas } & Masculino n (\%) & Femenino n (\%) \\
\hline Disnea & $206(89,57)$ & $77(77,78)$ \\
Tos & $173(75,22)$ & $68(68,69)$ \\
Malestar General & $142(61,74)$ & $73(73,74)$ \\
Fiebre & $137(59,57)$ & $68(68,69)$ \\
Dolor de Garganta & $64(27,83)$ & $22(22,22)$ \\
Cefalea & $47(20,43)$ & $21(21,21)$ \\
Diarrea & $26(11,30)$ & $10(10,10)$ \\
Dolor de Pecho & $16(6,96)$ & $12(12,12)$ \\
Otros & $20(8,70)$ & $6(6,06)$ \\
Dolor de Espalda & $7(3,04)$ & $10(10,10)$ \\
Congestión nasal & $12(5,22)$ & $2(2,02)$ \\
Disosmia & $2(0,87)$ & $3(3,03)$ \\
Disgeusia & $2(0,87)$ & $3(3,03)$ \\
Resultados de análisis de & & \\
laboratorio & & \\
Linfopenia <800 Cell/Ul & $123(53,48)$ & $45(45,45)$ \\
Proteína C Reactiva & $94(40,87)$ & $34(34,34)$ \\
LDH>350u/L & $84(36,52)$ & $32(32,32)$ \\
Ferritina $>700$ ng/ml & $37(16,09)$ & $14(14,14)$ \\
Dímero D $>1000$ ug/ml & $26(11,30)$ & $12(12,12)$ \\
Plaquetopenia & $24(10,43)$ & $13(13,13)$ \\
\hline
\end{tabular}

El tratamiento recibido por los pacientes hospitalizados que salieron de alta fue principalmente el oxígeno $89 \%$ y la enoxaparina $87 \%$, azitromicina $86 \%$ y ceftriazona $70 \%$ siguieron en importancia y metilprednisolona con hidroxicloroquina tuvieron la misma proporción $58 \%$ (Tabla 3).

Tabla 3. Tratamiento recibido durante la estancia hospitalaria de pacientes con diagnóstico de SarsCov2 hospitalizados en EsSalud. Región Lambayeque. abril-junio 2020.

\begin{tabular}{lc}
\hline Tratamiento & $\mathrm{n}(\%)$ \\
\hline Oxigeno (Cbn O Mr) & $293(89,06)$ \\
Enoxaparina & $286(86,93)$ \\
Azitromicina & $282(85,71)$ \\
Ceftriaxona & $230(69,91)$ \\
Metilprednisolona & $192(58,36)$ \\
Hidroxicloroquina & $191(58,05)$ \\
Paracetamol & $175(53,19)$ \\
Antiretrovirales & $149(45,29)$ \\
Ivermectina & $114(34,65)$ \\
Otro Antibiótico & $85(25,84)$ \\
Dexametasona & $79(24,01)$ \\
Metamizol & $17(5,17)$ \\
Warfarina & $9(2,74)$ \\
Intubación & $4(1,22)$ \\
\hline
\end{tabular}


Las complicaciones hospitalarias principales fueron la insuficiencia respiratoria $88,1 \%$ y la neumonía atípica $86,6 \%$ (Tabla 4 ).

Tabla 4. Complicaciones hospitalarias según género de pacientes con diagnóstico Sars-Cov2 hospitalizados en EsSalud en región Lambayeque. abril-junio 2020.

\begin{tabular}{lccc}
$\begin{array}{l}\text { Complicaciones } \\
\text { Hospitalarias }\end{array}$ & $\begin{array}{c}\text { Masculino } \\
\mathrm{n}(\%)\end{array}$ & $\begin{array}{c}\text { Femenino } \\
\mathrm{n}(\%) 99\end{array}$ & $\begin{array}{c}\text { Total } \\
\mathrm{n}(\%)\end{array}$ \\
\hline $\begin{array}{l}\text { Síndrome Distrés } \\
\text { Respiratorio Agudo (SDRA) }\end{array}$ & $207(90)$ & $83(83,8)$ & $290(88,1)$ \\
Neumonía Atípica & $204(88,7)$ & $81(81,8)$ & $285(86,6)$ \\
Anemia & $7(3,1)$ & $4(4,1)$ & $11(3,3)$ \\
Sepsis & $4(1,7)$ & $3(3,0)$ & $7(2,1)$ \\
Trombosis & $3(1,3)$ & $2(2,0)$ & $5(1,5)$ \\
Injuria Cardiaca Aguda & $1(0,4)$ & $2(2,0)$ & $3(0,9)$ \\
\hline
\end{tabular}

El tiempo promedio desde el inicio de síntomas hasta ingreso a emergencia fue 8,28 +/ - 3,62 días, la estancia en emergencia 2,28 +/- 1,65 días y la estancia en hospitalización $7,85+/-5,42$ días y la estancia en cuidados intensivos de 0,68+/- 1,30 días (Tabla 5)

Tabla 5. Tiempo promedio de estancias de pacientes con diagnóstico Sars-Cov2 hospitalizados en EsSalud región Lambayeque abril-junio 2020.

\begin{tabular}{lcc}
\hline Tiempo promedio & Promedio & Desviación estánsar \\
\hline De inicio de síntomas hasta & 8,28 & $\pm 3,62$ \\
ingreso a Emergencia & 2.88 & $\pm 1,65$ \\
Estancia en emergencia & 7.85 & $\pm 5,42$ \\
Estancia En Hospitalización &
\end{tabular}

\section{DISCUSIÓN}

Los pacientes hospitalizados y dados de alta con diagnóstico COVID 19 fueron en un $80 \%$ diagnosticados mediante prueba serológica lg $M$ dado que no ha existido cantidad suficiente de pruebas moleculares PCR en la región Lambayeque que solo diagnosticó el 3\% del total, y otro $17 \%$ lo fue mediante la tomografía de tórax con las lesiones características de vidrio esmerilado, sin embargo, tanto en China como Europa e incluso los casos de Lima fueron diagnosticados mediante prueba molecular.

Mejía F y colaboradores en una cohorte retrospectiva en el Hospital Cayetano Heredia analizó 369 historias: 241 (65,31\%) pacientes eran del sexo masculino y la mediana de edad era de 59 años ${ }^{(8)}$. Casas-Rojo et al realizó un "estudio de cohorte retrospectiva, multicéntrico, que incluye pacientes consecutivos hospitalizados con COVID-19 confirmada en toda España y hasta el 30 de junio de 2020 se incluyeron 15111 pacientes de 150 hospitales. Su mediana de edad fue 69,4 años y el 57,2\% eran hombres"(9). En China se informa por Wang en Wuhan en 138 pacientes hospitalizados $54,3 \%$ varones y una edad mediana de 56 $a_{n} \operatorname{si}^{(4)}$ y también por Wu et al estudio retrospectivo en 201 pacientes $63,7 \%$ masculino y la mediana de edad 51 años ${ }^{(7)}$.

En Chiclayo se encontró que los varones eran el $70 \%$ del total de casos y la media de edad fue 55,9+/-11 años, y el grupo de edad de 60 a más años era $41 \%$, sin embargo, adultos jóvenes de 30 a 49 años fueron 33\% la tercera parte de los casos.

Respecto a comorbilidades en los pacientes en el presente estudio la mayoría $70,5 \%$ las presentaban siendo tres las principales: Hipertensión arterial 25,84\% seguido de diabetes $18,54 \%$ y obesidad $13,68 \%$, en el Hospital Cayetano Heredia revisadas 369 historias de hospitalizados entre marzo y junio 2020 se encontró que el $68,56 \%$ presentaba al menos una comorbilidad dato similar al nuestro, sin embargo las comorbilidades tuvieron diferente orden de frecuencia: obesidad $(42,55 \%)$, diabetes mellitus $(21,95 \%)$ e hipertensión arterial $(21,68 \%)^{(8)}$, Llaro-Sánchez en los casos de fallecidos en Hospital Sabogal encontró como comorbilidades Hipertensión 30,43\%, obesidad 21,74\% y diabetes $17,39 \%^{(11)}$.

En China en un estudio de 41 pacientes Huang et al encontró que "menos de la mitad tenía enfermedades subyacentes: $32 \%$, incluyendo diabetes $20 \%$, hipertensión 15\% y enfermedad cardiovascular 15\%"(12), y por su parte Wu et al en Wuhan de un total de 201 pacientes $32,8 \%$ tenían comorbilidades: hipertensión $19,4 \%$, diabetes $10,9 \%^{(7)}$.

En el gran estudio español que al 30 de junio 2020 incluyó 15111 pacientes de 150 hospitales “se observó un elevado porcentaje de pacientes con comorbilidades (el 61,4\% tenía un índice de Charlson moderado o severo). Adicionalmente, el $16,5 \%$ de los pacientes tenían un grado moderado o severo de dependencia para actividades de la vida diaria (índice de Barthel inferior a 60). Las comorbilidades más frecuentes fueron hipertensión arterial (50,9\%), dislipemia $(39,7 \%)$, obesidad $(21,2 \%)$ y diabetes mellitus $(19,4 \%)^{(9)}$; en este informe, llama la atención el considerar como un factor de riesgo la dislipidemia que por lo general no se considera en otros estudios.

Los cinco signos y síntomas más frecuentes referidos por los pacientes COVID 19 en los hospitales de Essalud de Chiclayo fueron la disnea $86 \%$, tos $73 \%$, malestar general $65 \%$, fiebre $59 \%$ y dolor de garganta $26 \%$, en el Rebagliati Escobar y col. encontró fiebre y disnea en el $78,6 \%$, tos en el $71,4 \%$ y diarrea en el $28,6 \%^{(3)}$, en el Sabogal Llaro encontró en primer lugar disnea como en nuestro estudio $91,3 \%$, seguido de fiebre y tos $86,9 \%$, 
malestar general $43,4 \%$ y dolor de garganta $26 \%{ }^{(11)}$.

En China Wang et al encontró predominancia de fiebre $98,6 \%$, fatiga $69,6 \%$, tos $59,4 \%$, anorexia $39,9 \%^{(4)}$, por su parte Chen et al refiere en 99 pacientes que tenían fiebre $83 \%$, tos $82 \%$, dificultad para respirar $31 \%^{(14)}$ y Huang et. al. encontraron fiebre $98 \%$, tos $76 \%$, fatiga $44 \%^{(12)}$, Liu et al encuentra fiebre $81,8 \%$, tos $48,2 \%$ y fatiga $32,1 \%{ }^{(10)}$ y Wu et al señala fiebre $93,5 \%$, tos $81,1 \%$, disnea $39,8 \%$ y fatiga $32,3 \%^{(7)}$.

Casas-Rojo et al encontró en su revisión de 15111 pacientes en España que la fiebre 38 C o mayor fue $63,2 \%$, tos seca $58 \%$, disnea $57,6 \%$, astenia $43,6 \%$, diarrea $23,7 \%^{(9)}$ y en el estudio de casos y controles de pacientes sintomáticos compatibles con covid19 realizado en Francia Zayet encontró en el grupo de positivos por prueba molecular los síntomas más comunes fueron tos $79 \%$, cefalea $78 \%$, mialgias $75 \%$, fiebre $74 \%^{(13)}$.

Como se observa en los datos revisados son tres los síntomas más referidos: fiebre, tos y disnea sin embargo existen en la literatura otros síntomas que en su momento se consideraba como un signo de alerta por considerarse que podría ser el primer signo de inicio de síntomas: anosmia y disgeusia que en Francia estuvieron presentes en $63 \%$ y $65 \%$ de los positivos y que fue un elemento de gran diferencia con los que salieron negativos a los análisis pero tenían otros síntomas generales similares, además tenía una sensibilidad de $65 \%$, especificidad $84 \%$ y valor predictivo $76,5 \%{ }^{(13)}$, sin embargo en España solo se registró en 7,1\% la anosmia ${ }^{(9)}$ y en nuestro estudio solo estuvo registrado en $2 \%$ de los pacientes, puede ser que no se solicitaron a los pacientes información específica de esos signos que pudieran ser de gran importancia y requieren estudios de seguimiento en los nuevos casos que se confirmen positividad.

Los resultados de laboratorio que utilizados como marcadores de inflamación en los pacientes con diagnóstico de COVID 19 fueron señalados mediante la resolución ministerial 240-2020-MINSA en el Perú y se consideran en la actualidad: Linfopenia $<800 \mathrm{cell} / \mathrm{ul}$, Proteinca $C$ reactiva $>100 \mathrm{mg} / \mathrm{dl}$, Ferritina $>700 \mathrm{ng} / \mathrm{ml}$, Dimero $D>1000$ UG/M y Deshidrogenasa láctica > $350 u / /^{(15)}$.

Al respecto en Chiclayo destaca la linfopenia en el 51\%, Proteína C reactiva en 39\%, LDH >350u/L 35\%, Ferritina $>700 \mathrm{ng} / \mathrm{ml} 16 \%$, Dímero $\mathrm{D}>1000 \mathrm{uG} / \mathrm{ml} 12 \%$, plaquetopenia 11\%; en Lima Escobar encontró linfopenia en $64,3 \%{ }^{(3)}$ LLaro encontró linfopenia $39,1 \%$ y lactato deshidrogenasa elevado $34,78 \%{ }^{(11)}$. En España Casas-Rojo et al refiere los valores elevados de Ferritina $73,5 \%$, lactato deshidrogenasa $73,9 \%$, dímero D $63,8 \%$, linfopenia $52,8 \%{ }^{(9)}$.
Los estudios de China también precisan linfopenia $70,3 \%(4), 64 \%(7)$ lactatato deshigrogenasa elevado $39,9 \%(4) 68,2 \%(7)$, Liu $72 \%$ linfopenia ${ }^{(10)}$.

La citada RM 240-2020 también señala las pautas de tratamientos de pacientes en el Perú y los recibidos durante la hospitalización en Essalud de Chiclayo fue fundamentalmente el oxígeno $89 \%$, enoxaparina $87 \%$, antibióticos predominó azitromicina seguida de ceftriaxona, en corticoides se utilizó principalmente la metilprednisolona $58 \%$, la dexametasona $24 \%$, antiretrovirales $45 \%$.

En Lima Escobar refiere azitromicina 71,4\%, hidroxicloroquina $64,3 \%$, antibióticos de amplio espectro $57,1 \%{ }^{(3)}$ y LLaro indica que en el Sabogal fueron Hidroxicloroquina más azitromicina $39,3 \%$ y la hidroxicloroquina más azitromicina más un corticoide $39,3 \%$ lamentablemente sus pacientes fallecieron ${ }^{(13)}$.

La revisión española señala que el $85,6 \%$ recibió betalactámico, 71,7\%, Hidroxicloroquina, el $61,4 \%$ Lopinavir/ritonavir, azitromicina 60,8\%, corticoides sistémicos $35,2 \%$, heparina en dosis profilácticas $64,7 \%$ con un resultado final de fallecimientos del $20 \%{ }^{(9)}$.

En China los tratamientos fueron Oseltamivir $89,9 \%$ y moxifloxacina $64,4 \%$, azitromicina $18,1 \%$ y corticoides $44,9 \%{ }^{(4)}$, Oxigeno $82,5 \%$, metilprednisolona $30,8 \%$, antibiótico $97,5 \%$, antiviral $84,6 \%$.

La complicación más preocupante de la infección por SARS-CoV-2 (covid-19) es la insuficiencia respiratoria hipoxémica aguda que requiere ventilación mecánica. Los argumentos a favor y en contra de la intubación temprana aún no se resuelven, en gran parte porque faltan buenas pruebas. Dado que el Síndrome de Distrés Respiratorio Agudo (SDRA) causado por covid-19 es similar al SDRA con otras causas, los principios que sustentan la ventilación también deberían ser similares $^{(16)}$.

Las complicaciones hospitalarias que se refieren en España fue el SDRA 33,1\%, neumonía bilateral 66,3\% y neumonía unilateral $20,5 \%$, sepsis $6,2 \%$ que llevaron a un ingreso a UCI $8,3 \%$ y fallecimiento general $21 \%{ }^{(9)}$, en China Wang et al refiere SDA 61,1\%, shock 30,6\%, 26,1\% pasaron a $\mathrm{UCl}^{(4)}$ y Wu et al refieren infiltrado bilateral $95 \%$ y el SDRA $41,8 \%$, con ingreso a UCl $26,4 \%$ y fallecimiento $21,9 \%^{(7)}$. Mejía indica que el requerimiento de $\mathrm{UCl}$ fue de $10,10 \%$. Nosotros encontramos el SDRA en $88,1 \%$, neumonía $86,6 \%$, anemia $3,3 \%$, sepsis $2,1 \%$ y trombosis $1,5 \%$.

Los tiempos promedio de estancia hospitalaria fue de 7 días (rango 3-10 días) según Mejía y col en Lima ${ }^{(8)}$, Wang et al encontró mediana de estancia 10 días (rango entre 
7-14 días) ${ }^{(4)}$, Wu et al refiere 13 días (rango 10-16 días) ${ }^{(7)}$, nosotros encontramos una estancia en hospitalización promedio de 7,85 días $+/$ - 5,42 días, aunque también se estimó la estancia en emergencia promedio de 2,88 +/ 1,65 días que es importante resaltar pues la realidad es que existe demora en internar a los pacientes por falta de camas hospitalarias.

Los pacientes de Chiclayo salieron de alta recuperados uno de cada cinco $(21,8 \%)$, la gran mayoría fueron considerados como recuperados parcialmente $72,64 \%$ y algunos ya tenían secuelas 5,78\%.

A la fecha de redacción de este artículo 23 de setiembre 2020 se contabilizaban a nivel mundial ya un total de 31 375325 casos confirmados y 966399 fallecimientos, el Perú figura en quinto lugar mundial de incidencia con $772896 \operatorname{casos}^{(17)}$. Lamentablemente también se registra oficialmente 31474 fallecidos y considerando el dato oficial de INEI de población estimada para el Perú en el año 2020 de 32625948 habitantes tenemos la más alta tasa de mortalidad en el mundo: 9647 x 100000 habitantes y una alta tasa de letalidad de 4,07 por 100 $\operatorname{casos}^{(18)}$, lo que indica la gran importancia de continuar realizando estudios sobre diferentes aspectos de la situación de la pandemia en nuestro país y región.

El estudio tiene como principal limitación son los errores de registro o ausencia de datos en los registros respectivos.

Se recomienda realizar estudios analíticos para precisar factores de riesgo de mortalidad en nuestro medio para los pacientes con Covid19 y seguimiento de las altas para precisar la frecuencia y tipo de secuelas que pueden existir.

Se concluye que la gran mayoría de pacientes hospitalizados en el Seguro Social del Perú en la región Lambayeque fueron varones, promedio edad 55, 9 años, disnea, tos, malestar general y fiebre fueron los principales síntomas y en laboratorio predominó la linfopenia con proteína $\mathrm{C}$ reactiva y $\mathrm{LDH}$ altas, con tratamientos en base a oxígeno, enoxaparina, antibióticos y corticoides y un promedio de estancia en hospitalización de 7,85 días.

Agradecimiento: Al Dr. Jaime Salazar Zuloeta, Jefe de Departamento de Medicina II del Hospital Nacional Almanzor Aguinaga Asenjo, por las facilidades brindadas para realizar la presente investigación.

Conflictos de interés: Los autores niegan conflictos de interés.

Financiamiento: Autofinanciado.

\section{REFERENCIAS BIBLIOGRÁFICAS}

1. Lu H, Stratton CW, Tang Y-W. Outbreak of pneumonia of unknown etiology in Wuhan, China: The mystery and the miracle. J Med Virol. 2020;92: 401-402.

2. Pérez -Abreu MR, Gómez- Tejeda JJ, Dieguez Guach RA. Características clínico-epidemiológicas de la COVID-19. Rev haban cienc méd. 2020; 19(2):e_3254.

3. Escobar G, Matta J, Taype W, Ayala R, Amado J. Características clínico epidemiológicas de pacientes fallecidos por covid-19 en un hospital nacional de Lima, Perú. Rev. Fac. Med. Hum. 2020; 20(2):180-185.

4. Wang D, Hu B, Hu C, Zhu F, Liu X, Zhang J et al. Clinical Characteristics of 138 Hospitalized Patients With 2019 Novel Coronavirus-Infected Pneumonia in Wuhan, China. JAMA. 2020;323(11):1061-1069.

5. MINSA: Casos confirmados por Coronavirus Covid19 ascienden a 285213 en el Perú (Comunicado $N^{\circ}$ 153) [Internet]. plataforma digital única del Estado Peruano. 2020 [citado 6 junio 2020]. D i s p o n i b l e e n : https://www.gob.pe/institucion/minsa/noticias /189369-minsa-casos-confirmados-porcoronavirus-covid-19-ascienden-a-285-213-en-elperu-comunicado-n-153

6. Gerencia Regional de Salud Lambayeque. Reporte COVID19 [Internet]. Situación al 21 de junio 2020 a las 12.00 horas [citado 22 junio 2020]. https://www.facebook.com/GERESALAMB/photo s/3256668021050673

7. Wu C, Chen X, Cai Y, Xia J, Zhou X, Huang H. et al. Risk Factors Associated With Acute Respiratory Distress Syndrome and Death in Patients With Coronavirus Disease 2019 Pneumonia in Wuhan, China. JAMA Intern Med 2020;180(7):934-943.

8. Mejia F, Medina C, Cornejo E, Morello E, Vasquez S, Alave J, Schwalb A, Malaga G. Características clínicas y factores asociados a mortalidad en pacientes adultos hospitalizados por COVID-19 en un hospital público de Lima, Perú. [Internet] 2020(citado may 2).disponible en :

https://preprints.scielo.org/index.php/scielo/p reprint/download/858/1187/1244

9. Casas-Rojo J.M. Antón-Santos J. Millán-NúnezCortés C. Lumbreras-Bermejo J.M. Ramos-Rincón E. Roy-Vallejo A. et al. Características clínicas de los pacientes hospitalizados con COVID 19 en España: resultados del registro SEMI-COVID 19. Rev Clin Esp. 2020.

10. Liu K, Fang YY, Deng Y, Liu W, Wang MF, Ma JP. Et al. Clinical characteristics of novel coronavirus cases in tertiary hospitals in Hubei Province. Chin Med J 2020;133(9):1025-1031.

11. Llaro-Sánchez MK, Gamarra-Villegas B, CamposCorrea K.E. Características clínicoepidemiológicas y análisis de sobrevida en fallecidos por COVID-19 atendidos en 
establecimientos de la Red Sabogal-Callao 2020. Horiz Med (Lima) 2020; 20(2): e1229.

12. Huang $\mathrm{C}$, Wang $\mathrm{Y}$, Li X, et al. Clinical features of patients infected with 2019 novel coronavirus in Wuhan, China [published correction appears in Lancet. 2020 Jan 30]. Lancet. 2020; 395(10223):497-506.

13. Zayet S, Klopfenstein T, Mercier J, Kadiane-Oussou NJ, Cheong-Wha LL, Royer PY. Contribution of anosmia and dysgeusia for diagnostic of COVID-19 in outpatients. [Internet]. Infection published on l i n e $\quad 14 \quad \mathrm{ma} y \quad 2020$. https: / / doi.org/10.1007/s15010-020-01442-3

14. Chen N, Zhou M, Dong X, Qu J, Gong F, Han Y. Epidemiological and clinical characteristics of 99 cases of 2019 novel coronavirus pneumonia in Wuhan, China: a descriptive study [Internet]. china: The Lancet; 2020 [citado 8 mayo 2020]. Disponible en: https://doi.org/10.1016/ S01406736(20)30211-7

15. Ministerio de Salud. Resolución ministerial 2402020, 29 de abril [Internet]. 2020 (citado el 3 de mayo del 2020) disponible en: https://cdn.www.gob.pe/uploads/document/fil e/668370/RM_240-2020-MINSA.PDF.

16. INTRAMED. Insuficiencia respiratoria por Covid 19
[Internet]. (citado el 20 de mayo 2020). Disponible en:

https://www.intramed.net/contenidover.asp?co ntenidoid $=96064$

17. World Healtn Organization. WHO Coronavirus Desease (COVID 19). [Internet]. Dashboard Data last updated: 2020/9/23, 12:21pm CEST available i

https: / / covid19. who.int/?gclid=CjwKCAjw5Kv7B RBSEiwAXGDEIWB9iL4KmFpegewM33xbksYmyX90 OS0-8xfZrrT jsUXCQh1zmSemRoCZLYQAvD BwE

18. Jhon Hopkins. Coronavirus resource center. Cases and mortality by country. [Internet]. 2020/9/23. D i s p o n i b l e e $n$ : https://coronavirus.jhu.edu/data/mortality.

\section{Correspondencia}

Rosario Alexandra Soto-Cáceres Cabanillas.

Dirección: Rentema 570 Urb. La Primavera Chiclayo.

Teléfono: 924186649

Correo: ralex264@hotmail.com

\section{Revisión de pares}

Recibido: 15/09/2020

Aceptado: 15/12/2020 